\title{
Artificial intelligence in fifth generation computers
}

\author{
R NARASIMHAN \\ Tata Institute of Fundamental Research, Homi Bhabha Road, Bombay \\ 400005 , India
}

\begin{abstract}
Artificial inielligence forms an intrinsic aspect of the competence of fifth generation computers in view of the need for them to be primarily knowledge processing systems. We analyse in this paper how this need for knowledge processing arises. In the main body of the paper various issues that arise in knowledge representation and use are discussed. The point of departure for these discussions is the recent work on expert systems. We point out the difference between tacit and propositionizable knowledge and argue the need for modelling tacit knowledge also in fifth generation computers.
\end{abstract}

Keywords. Artificial intelligence; fifth generation computers; knowledge processing; expert systems; heuristics.

\section{Introduction}

The defining characteristic of fifth generation computers (FGC) is that they are to be knowledge processing machines in contrast to being merely data processing machines as is the case with most of the present generation computers. The need for artificial intelligence (henceforth AI) in FGC fundamentally arises in trying to come to grips with this knowledge processing aspect. When formulating their own FGC Project the Japanese were very explicit about this central design issue: What is the appropriate hardware architecture and related machine language level basic software, so that knowledge processing can be handled in a natural way?

Of the many possible choices that, in principle, could have been made, the Japanese have opted for a particular one, namely to build the FGC as a predicate logic machine, that is, as, an inference machine. The machine language is to be a predicate logic language-some extended version of PROLOG. "The hardware will be basically designed for parallel operation and associative search since its basic function will be inference. Present day yon Neumann type computers are basically built for sequential operation and address search. Therefore, a parallel, associative inference machine will be a sort of non-von Neumann type computer" (Fuchi 1984, p. 1). Since what is considered to be a very high-level language in current computer technology would become the machine language of the new generation machines, "the capability of the language will be utilized to achieve sophisticated functions such as knowledge information processing and natural language processing" (Fuchi 1984, p. 1).

It is important to keep clearly in view the central aim of the Japanese FGC Project and 
distinguish it from the particular design decisions that have been made by them to implement it. While there is general agreement about the need to work towards achieving that aim, the specific design decisions have generated some controversy (see, for example, Bobrow 1984, p. 138). We shall restrict our discussion in what follows in this paper to the central aim of the FGC Project.

Our principal concern here is to analyse the need for knowledge processing as a central capability in the next generation computers. From a consideration of the kinds of task environments and man-machine interactions that have to be coped with, we shall identify in the next section the Al research areas that underpin the realization of machines with the requisite capabilities to deal with these problem domains. It will be seen that in each one of these problem domains knowledge processing is of central concern.

Three principal issues that relate to knowledge processing are knowledge representation, control and use of knowledge, and acquisition of knowledge. Of these, the knowledge acquisition aspect is the least understood and remains very much an open problem. The aspects that have received the most attention are knowledge representation and use - especially in the context of design of expert systems. In \$3 we shall briefly discuss the status of work in knowledge engineering and knowledge representation and the methodologies that have been intensively studied.

In \$4 we shall discuss some of the problems that arise in the control and use of knowledge. We shall be specifically concerned with the role of heuristics in knowledge usage when confronted with particular problem situations. Our discussion will be based essentially on the work of Lenat (1982) on 'heuretics', i.e., theory of heuristics.

All the work in Al on expert systems is predicated on the propositionizability of knowledge. We shall argue that tacit knowledge is equally important to model especially in enhancing the man-machine interaction capabilities of FGC. Section 5 is a summary of the main arguments and discussions of the paper.

\section{Need for knowledge processing : Defining the AI capabilities}

\subsection{Limitations of present-day computers}

Computers, especially after the advent of microcomputers based on microprocessor chips, have become highly pervasive information processing engines underpinning a wide variety of activities. The daily living and work environments of an increasing number of people are beginning to be endowed with more and more intelligence through the deployment of equipment and tools that are controlled by programmed or programmable microprocessor chips. The potential for productivity-increase arises out of the availability of access to such intelligent supports in the work environment. Nevertheless, the impact of computers, in general, has been far less than what one would have expected considering their intrinsic potential. There are two principal reasons for this:

i) the software bottleneck

ii) the poverty of the communication interface between computers and their environments, especially human beings.

The software bottleneck stems from the fact that piesent-day computers function typically as mechanical slaves. They execute programmed action sequences in a 
mechanical way. Programming involves the explicit specification of all the action steps needed to solve a problem, providing alternatives for all contingencies that may be encountered on the way. This programming, moreover, has to be carried out in a rigidly constrained programming language. The consequence of these requirements is the need for a large number of highly skilled and specially trained individuals who can analyse the task on hand, formulate the needed programs, and implement them in one of a variety of specialized programming languages.

Programming, therefore, is a high-quality, manpower-intensive task. The software bottleneck develops because the requisite manpower cannot be developed fast enough to meet the ever enlarging programming needs. One way out is, of course, to partially automate this task through appropriate intelligent tools. In fact, one of the important projected application areas of FGC is precisely this.

In current computer practice the interaction between humans and computers is restricted to a set of simple channels. Input to a computer is via keyboards, graphic screens with pointer-attachments of one kind or another, and specialized character readers and sensors. Output from a computer is through printers, plotters, and graphic screens. The intrinsic poverty of these channels can be appreciated by contrasting these with the following modalities of normal communication between human beings: i) speech ii) documents - handwritten, typewritten, printed, iii) pictorial information sketches, charts, drawings, maps, photographs, iv) 3-D models.

The two kinds of limitations discussed above prevent, at present, the wide deployment of computers in a variety of important task environments, where increases in efficiency and productivity could be extremely valuable. The following is a sample list of such task environments.

\subsection{Some typical task environments}

1. The computer as an informed office assistant:

- produce a typescript from dictation,

- edit a document from roughly specified instructions (spoken/written),

- make a typescript of a handwritten document,

- make an abstract of a document

- retrieve filed information based on roughly specified requirements,

- plan a travel itinerary satisfying given constraints.

2. Computer as a knowledgeable teacher:

- take a student through a graded sequence of lessons,

- interact with a student to clarify doubts, correct misunderstandings,

- administer tests to evaluate understanding, knowledge transfer,

- grade a batch of students.

3. Computer as an expert consultant:

- medical diagnosis and advice,

- equipment maintenance and repair,

- configuring equipment to engineer a system with specified performance,

- assistance in planning,

- assistance in detailed design.

4. Computer as a free agent (robotics):

- manipulating objects,

- assembling equipment from a kit, 
- navigating, exploring,

- acting as an informed shop-floor assistant.

\subsection{Al capabilities needed}

In the last two decades research workers in AI have tried to tackle some of the tasks listed above. Except possibly for a few expert systems - addressing the tasks in area (3) above - that have been successfully demonstrated, it is fair to say that none of the tasks listed above is within the competence of present-day computer technology. The distinguishing characteristic of each one of these tasks is that to grapple with it successfully one has to be intelligent and well-informed (i.e., knowledgeable). In addition, one must be endowed with complex sensory-motor capabilities. Lastly, ability to communicate in a natural language is a pre-requisite for interaction with/between humans in all these task environments.

Intelligent behaviour: 1) uses knowledge; (2) involves judgment, and (3) exhibits understanding. These three are key issues in building systems that can function in a task environment intelligently and in an informed manner.

The knowledge that is needed is of several kinds. For instance,

- Knowledge of the world (common-sense knowledge),

- knowledge of the task domain (technical/professional knowledge),

- knowledge of the immediate interaction situation (contextual knowledge),

Judgment is the capability to gather relevant evidence, evaluate, and draw conclusions.

In trying to understand something, for instance, a spoken utterance (speech); a picture, drawing, or a photograph of a scene (vision); a problem or a puzzle, we bring to bear on the task a variety of intellectual competences. An illustrative list of such competences is:

- pattern matching, classification, analysing a complex into simples,

- naive set-theoretic notions, e.g., all, none, at least one, at most one, etc.,

- naive arithmetic notions, e.g., add, subtract, count, ordering etc.,

- making inferences from available knowledge,

- making conjectures and judging their validity in given situations.

We can now summarize the AI capabilities that computer systems must be endowed with before tasks of the kind listed earlier can be meaningfully attempted. These fall into two broad categories.

1. Enlarging the input-output modalities of interaction with the environment

- speech,

- vision,

- touch,

- manipulation (hand),

- locomotion,

2. Enlarging the cognitive capabilities

- planning, problem-solving-using a knowledge-base with judgment and understanding, 
- learning -skill-learning; acquiring a knowledge-base; modifying an available knowledge base from experience,

- language use -interaction capability in a natural language.

There exists a vast and rapidly growing literature-base in AI dealing with all these areas. This should be consulted to get a proper feel for the kinds of issues that arise in dealing with any of the aspects listed above. As noted earlier, we shall restrict our discussions in the rest of this paper to one specific research area: planning and problemsolving using a knowledge-base with judgment and understanding. To this end, we shall consider next the work done on expert systems.

\section{Issues in knowledge processing}

\subsection{Two kinds of knowledge}

It is useful to group knowledge into two broad categories as indicated in table 1.

This division of knowledge into two categories has significance and value in accounting for behavioural pragmatics. Literacy (i.e., writing, reading, the use of notations and symbols), in general, is a prerequisite for the acquisition of knowledge that is propositionizable. This is not the case for the acquisition of tacit knowledge. We shall further discuss this topic in a later section. The two kinds of knowledge are, however, interdependent in the sense that one's perceptual-motor competence may be modified by one's professional knowledge. An expert may (and quite often does) see the world differently from the way a layman does.

AI methodologies developed in the last two decades all stem from attempts to simulate human performance based on 'professional' knowledge, i.e., on propositionizable knowledge. Currently available AI techniques work best when,

1. the problem domain is well-delimited,

Table 1. The two kinds of knowledge

\begin{tabular}{|c|c|c|}
\hline & \multicolumn{2}{|c|}{ Knowledge that is } \\
\hline & tacit & propositionizable \\
\hline $\begin{array}{l}\text { Underpins what } \\
\text { behaviour? }\end{array}$ & $\begin{array}{l}\text { 1. Perceptual-motor competence } \\
\text { 2. Skill acquisition and use }\end{array}$ & $\begin{array}{l}\text { 1. Puzzles, games, defined through } \\
\text { explicit rules } \\
\text { 2. Problem-solving in an articulated task } \\
\text { domain }\end{array}$ \\
\hline How acquired? & $\begin{array}{l}\text { Through informal means: } \\
\text { 1. exposure to examples } \\
\text { 2. apprenticeship } \\
\text { 3. rehearsal and practice }\end{array}$ & $\begin{array}{l}\text { Through formal means: } \\
\text { 1. systematic formal tuition } \\
\text { 2. learning based on theories } \\
\text { 3. text-books }\end{array}$ \\
\hline What is it called? & $\begin{array}{l}\text { 1. Common-sense knowledge } \\
\text { 2. Craft knowledge }\end{array}$ & 1. Professional or expert knowledge \\
\hline Who has it? & $\begin{array}{l}\text { Everybody (artisans, craftsmen when } \\
\text { skill-based) }\end{array}$ & Professionals (experts) \\
\hline
\end{tabular}


2. the problem domain knowledge is explicitly and comprehensively propositionizable,

3. this knowledge can be organized in a readily usable form, and

4. the knowledge so organized can be used to converge rapidly to plausible solutions from initial problem-statements.

\subsection{Expert systems: scope of work}

Expert systems are engineered systems that try to exploit the strength of the AI methodology through the use of the following strategy:

1. choose a well-delimited application area of practical value,

2. propositionize the knowledge of this area,

3. organise this knowledge in a usable form, and

4. work out strategies to use the knowledge to go from typical problem statements to plausible solutions.

Steps 2 and 3 constitute knowledge-engineering; step 4 constitutes inference engineering. The basic structure of an expert system can be schematized as shown in figure 1 .

Using this approach, a large number of expert systems have been designed during the last decade. A representative sample is shown in table 2. For more information about these systems the original sources must be consulted (see Stefik et al 1982) for references.

As one might expect, analysis is a far more straightforward problem for expert systems to handle than synthesis. The most successful implementations are in diagnosis and monitoring. By contrast, planning, theory construction, design and so on, have proved to be very much more difficult to automate using knowledge-based systems. For a good survey of work on expert systems see Duda \& Shortliffe (1983).

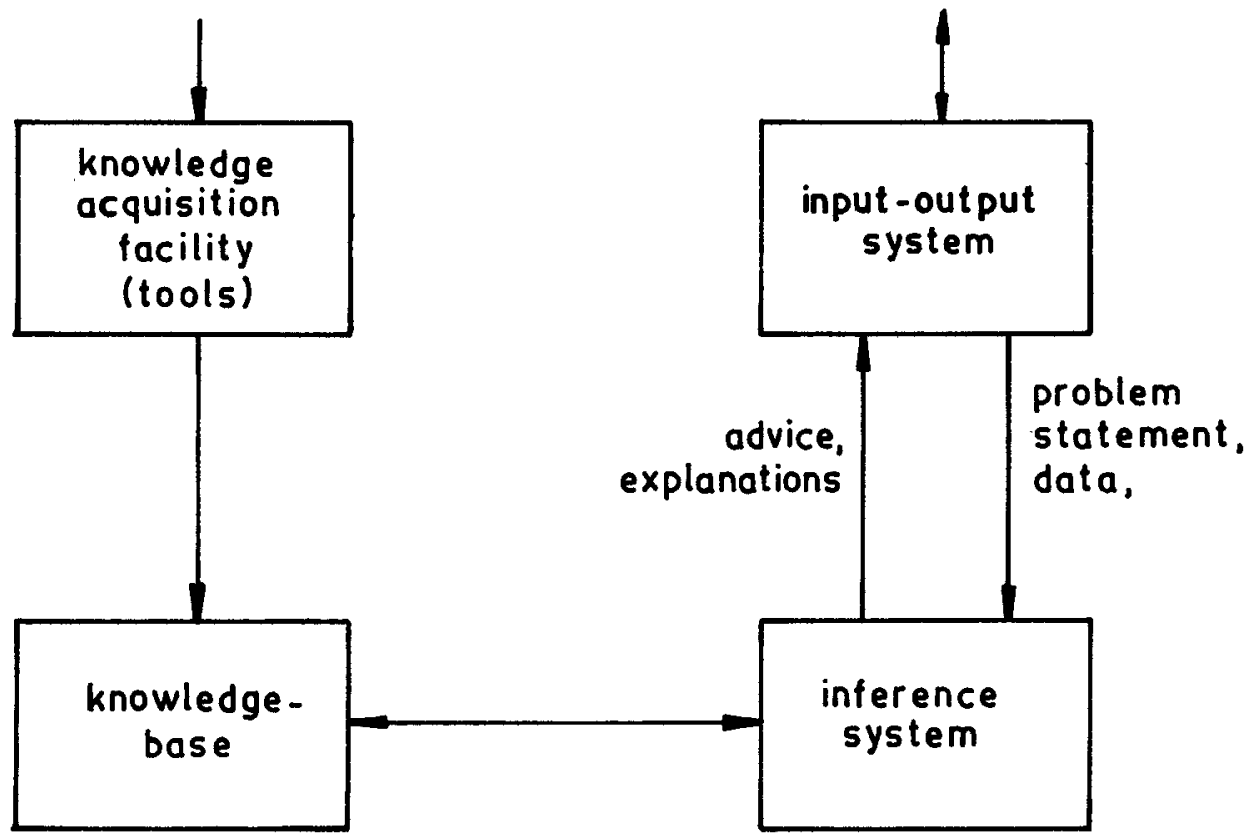

Figure 1. Basic structure of an expert system. 
Table 2. Examples of expert systems successfully demonstrated

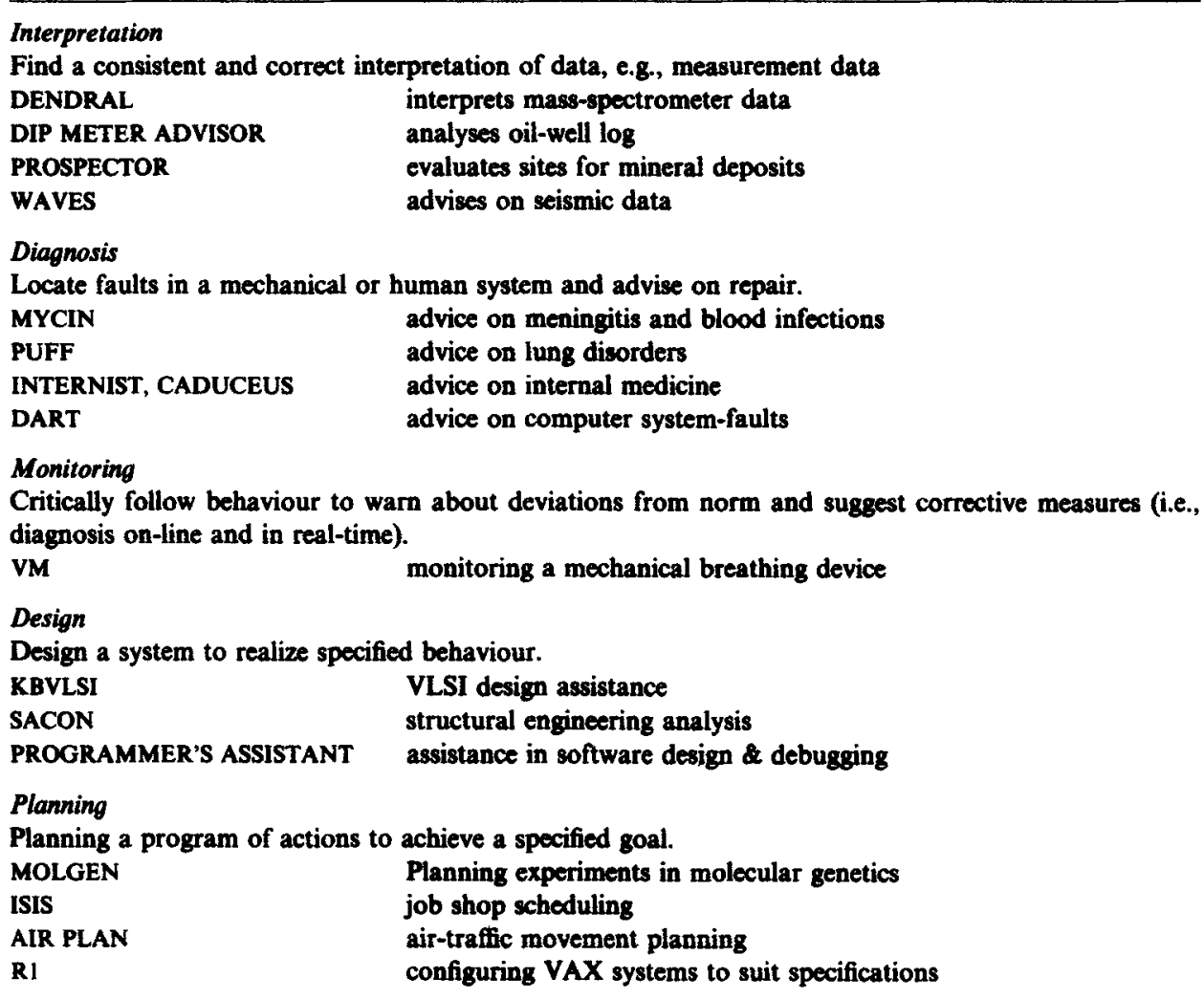

\subsection{Expert systems: design details}

In AI consultation systems there is a deliberate separation of: 1. domain-specific knowledge-bases, 2. general mechanisms of evaluation, and 3. control strategies. In practically all experts systems so far implemented, the domain-specific knowledge is encapsulated as Rules with the following format:

if LHS then RHS (LHS $\rightarrow$ RHS), where LHS specifies a set of conditions or patterns for the rule to become applicable, and RHS specifies a resultant action, output, or inference that can be made - usually with some associated probability or weight. A typical system may have several hundred rules. To improve efficiency in access to, and in threading through, this knowledgebase, the rules must be grouped into larger knowledge chunks. Partitioned semantic nets, frames, or other grouping techniques have been utilized in practice.

To illustrate these ideas by means of a concrete implementation we can look at MYCIN (Shortliffe 1976). MYCIN is an expert system developed at Stanford University to assist physicians in the selection of antibiotics for patients with severe infection. Here, the knowledge-base is one large chunk consisting of over 500 rules of the form

if $P$ then $A$, where $P$ is a conjunction of predicates over triples (attribute, object, value), and $A$ is an assignment of value to some specific attribute. 
A typical, often-quoted example of a rule from MYCIN expressed in English is:

If the infection is meningitis, and, organisms were not seen in the stain of the culture, and, the type of infection may be bacterial, and, the patient has been seriously burned,

then there is suggestive evidence that Pseudomonas aeruginosa is one of the organisms that might be causing the infection.

The control structure used in MYCIN is goal directed backward chaining.

1. At any given time MYCIN is trying to assign a value to an attribute.

2. To do so, it retrieves all the rules whose RHS $(A)$ relates to this attribute value assignment.

3. It chooses one of these and evaluates its LHS $(P)$. While doing so, if some attribute value is needed and not known, it sets up a subgoal and goes to (2).

4. If no relevant rule provides the needed information, it generates a query to the user.

5. If the query is not answered, it neglects the concerned rule and goes to (3).

6. The effort is terminated based on pregiven termination conditions.

The user can query MYCIN about why a question was asked, or how a conclusion was reached. MYCIN responds by providing a trace of the rule chain it has traversed upto the concerned point. This ability to provide an explanation of one's behaviour is extremely important in an expert system for sustaining credibility.

To illustrate the use of a knowledge-base that is more structured and ramified, we might look at INTERNIST where the domain of consultation is internal medicine (Pople 1977, p. 1030). The knowledge-base consists of disease entities and manifestations (history items, symptoms, physical signs, laboratory data). About 400 disease items are encoded in the knowledge-base with over 2000 manifestations.

With each disease entity is associated a list of manifestations with weights indicating the frequency of occurrence. With each manifestation is associated a disease list where this manifestation occurs, again with a weight. Disease entities are grouped into a hierarchy, the top-most being organ-associated, e.g., liver-disease, kidney-disease, etc. The bottom-most nodes are disease entities. Other relations are defined over the above set to represent causal, temporal and other associations between disease entities. Relations are also defined over the set of manifestations, e.g., derivability of one from another or a set of others.

A hypotheses generator configures a search space in the form of an AND-OR graph at the disease-category level. Using the importance and frequency of occurrence of the disease-manifestation associations, the graph is pruned by stages.

\subsection{Aids to knowledge-engineering}

Perhaps the most difficult effort in building an expert system is the articulation and encapsulation of domain-knowledge. Typical knowledge-bases for practical applications may consist of $10^{3}$ to $10^{5}$ If - Then rules at the present state of the art. Typical estimates given for putting together a knowledge-base are 1-5 rules per hour. Building an expert system from scratch, hence, may take from several man-months to several man-years depending on the complexity of the task environment and the productivity of the tools available for knowledge engineering.

It is not surprising, therefore, that the design of computer aids to knowledge 
engineering has attracted much attention in AI research. An approach that has been tried with success is to build expert system skeletons with which an expert can interact in a controlled manner and convert his domain expertise into a knowledge-base by successive refinements.

A typical example of such a supportive system for knowledge engineering is TEIRESIAS (Davis 1977, p. 321). The basic design tactic is to clearly separate the control structure from the knowledge-base, and debug the control structure once for all. Also, the format for knowledge representation is pregiven and fixed, i.e., how knowledge is represented in the system is fixed. What knowledge the system is to use is then provided by the interacting expert (to conform to the pregiven format). The expert sets the initial conditions and observes the diagnostic behaviour of TEIRESIAS (T). T, based on the inputs provided uses the available knowledge to ask questions and provide diagnosis, i.e., make assertions. The expert can probe the system behaviour to critically look at why specific questions are asked, or why specific assertions are made. The traces for analysing such behaviour is provided by $T$ itself, as we saw earlier in the case of MYCIN. The expert, through such interactions, can enlarge, modify, or refine the knowledge he has already provided to the system stepwise till satisfactory convergence to adequate performance is achieved.

Other systems that have been constructed to provide similar assistance in creating domain-specific knowledge-bases through interaction are EMYCIN (van Melle 1979, p. 923) and AGE (Nii \& Aiello 1979, p. 645).

\subsection{Open problems}

All implemented systems represent knowledge in a uniform way as pattern-action rules. There have been a few attempts to design expert systems in the logic programming framework (see, for example, Clark \& McCabe 1982, for references). But our general criticism applies to this framework also. Clearly, a multiplicity of knowledge representations are needed for viable performance, to judge from the way human experts function. Knowledge can be encapsulated in a variety of ways as follows:

1. rules (statements, formulae, strings, etc.).

2. pictures (shapes, features, configurations, etc.),

3. schemata (charts, tables, maps, chemical diagrams, etc.).

Many open problems have to be solved before expert systems handling multiplyrepresented knowledge-bases can be designed. Some of these questions are:

1. what representation is best for what kind of knowledge,

2. how to move from one representation to another.

Another set of open problems relate to knowledge at the meta-level, i.e., knowledge about knowledge. Clearly, knowledge at this level is what is implicit in the control structure, in determining effective ways to search through available domainknowledge, in deciding what is the next step to take, in being able to profit from experience in using available knowledge. A set of questions that define problems at this level are the following:

1. how to reason about available knowledge;

2. what are good strategies for guessing; 
3. how to reason in a dynamic context, i.e., about situations that change with time (e.g., a robot in a real world);

4. how to revise one's beliefs based on new knowledge;

5. how to consolidate, abstract, schematize knowledge based on feedback from performance and/or interaction with an expert.

Much less work has been done on meta-knowledge than on domain knowledge. We shall discuss briefly in the next section some of the more important ideas and issues in this area.

\section{Issues in knowledge usage: The role of heuristics}

\subsection{Need for heuristics}

Given a knowledge-base of relevance to a task-domain, when confronted with a specific problem situation in this task-domain, what are good strategies for threading through the knowledge-base to decide on suitable actions to perform? It is often argued in the AIliterature that "in the knowledge lies the power", and the details of the control structure are not crucial. In other words, if knowledge is complete enough, even simple-minded search procedures would do.

If by 'knowledge' one means just knowledge of 'facts', clearly, in this extreme form the argument cannot be true. For, take the case of an axiomatized system, and consider the problem of determining whether a given assertion is a theorem or not. All the knowledge is in the axioms. Nevertheless, automatic theorem-provers demonstrate that solving this problem, in general, could become intractably complex. The issue, therefore, is not whether the knowledge-base is complete. What is important is that the knowledge should be represented such that it is effectively available for use.

As we saw earlier, in most implemented expert systems, knowledge of the problemdomain is encapsulated in the form of pattern-action rules. It is easy to see that in this format a good deal of the control structure is implicitly embedded in the knowledgebase. For, if the "patterns" are richly ramified to cover the varieties of problem situations likely to be encountered in the task-domain, given a particular situation, if pattern extraction is not an issue (which is the case in most of the expert systems implemented), determining which rules are relevant and which ones are not tends to become fairly trivial.

We can restate the same argument in a somewhat different way as follows. Taskdomain-dependent expertise can be based on three different kinds of foundations:

1. the availability of a rich and ramified enough theory which accounts for all the situations that arise in a task-domain (e.g., highly developed theory-based scientific domains);

2. the availability of a rich and ramified enough experiential background (e.g., a master chess-player);

3. an adequate theoretical background complemented by a rich enough experiential base (e.g., medical practice).

An expert, in all these cases, can structure his/her knowledge-base in a predigested form as a complex of pattern-action rules. In such circumstances, very little control structure is needed to apply one's knowledge. We can say that the expert brings to bear 
on the problem situation a rich "intuition" concerning the task-domain. The current technology of expert systems tends to model this kind of expertise.

It is easy to see that this approach will not be adequate to model "creativity"; that is, where it is necessary to build task-domain-dependent knowledge-bases and not merely apply an already built-up knowledge-base. The knowledge-base that is needed to accomplish this task is one at a meta-level; one that would provide guidance in exploring, experimenting and profiting from experience.

Such a knowledge-base would provide guidance precisely in answering the five questions that we posed at the end of the last section. Meta-level knowledge tends to be of value even to experts (or expert systems) in given task domains. This is so because the task-domain-dependent knowledge-base, even if encoded in the form of patternaction rules, is seldom complete, or exhaustive, or fully validated. Even an expert has to guess, experiment, correct himself and modify or re-encapsulate his existing knowledge-base. Heuristics are one set of aids to accomplish these tasks.

\subsection{Theorizing about heuristics}

A heuristic is a piece of knowledge that suggests, in a given problem situation, what actions are likely to lead to results and, hence, may be followed, and what actions are not likely to do so, and, hence, should be avoided. As we saw above in the context of discovering new knowledge about a domain, heuristics provide guidance in fruitful exploration, experimentation and evaluation of newly discovered concepts, relations, etc. In the context of applying already compiled domain knowledge, heuristics provide guidance in coping with uncertainties, dead ends, stalled moves, etc. They help also in profiting from past experience in order to modify, restructure, and otherwise refine available knowledge.

Lenat's work on AM and EURISKo provides, perhaps, the most substantial foundation to date for theorizing about heuristics. He himself has discussed at great length the nature of heuristics based on his experience with AM and EURISKO. His paper (Lenat 1982) should be consulted for a detailed background to the topic and for additional references. (Two other papers which provide valuable background information and insightful comments are a criticism of the AM work by Ritchie \& Hanna 1984, and a reply to this criticism by Lenat \& Brown 1984.) Here we shall summarize some conclusions from his discussion in order to provide an entry into the subject.

AM was a LISP program whose domain was elementary finite set theory. Its task was to generate empirical data, look for regularities in them and create new concepts. AM was initialized with 115 set theory concepts (sets, lists, ..., union, composition, ...) and 243 heuristics for proposing new concepts, generating data about them, evaluating them for 'interestingness', and so on.

In one hour of DEC-10 cpu run, AM generated 200 new concepts about half of which were judged to be reasonable. Concepts discovered by AM included: natural numbers, results such as de Morgan's laws, elementary divisibility theory, the fundamental theorem of arithmetic, etc.

EURISKO was a generalization of AM to test whether the same discovery procedure would work equally well in discovering new heuristics. Initial heuristics, instead of being hand-coded into rigid, unanalysable programs, were themselves represented in exactly the same way as 'functions' in AM. With this modification, heuristics, which 
were earlier applicable to mathematical functions, now became applicable to heuristics themselves. For example, in the following three heuristics, $f$ can be a mathematical function or another heuristic - in fact, it could be the very same heuristic.

$\mathrm{H}_{1}$ : If the results of performing $f$ have always been numerous and worthless, then lower the expected worth of $f$.

$\mathrm{H}_{2}: I f$ the results of performing $f$ are only occasionally useful, then consider creating new specialization of $f$ by specializing some slots of $f$.

$\mathrm{H}_{3}$ : If a newly synthesized concept has slots that coincide in value with those of an already existing concept, then the new concept should be destroyed because it is redundant.

'Slots' in the above refer to attribute-value pairs as provided in a Property List in LISP. More abstractly, slots can be thought of as parametrizations of an entity (i.e., a concept, a function, an operator, or a heuristic). Richness of parametrization, in fact, is the clue to an efficient discovery process. For, the three fundamental schemes that aid discovery are: specialization, generalization, and analogizing. All these are operations defined in terms of the parameter-sets.

Most heuristics are naturally stated as rules having the format

$$
\text { if }<\text { conditions }>\text { then }<\text { actions }>\text {. }
$$

A heuristic, instead of having simply an if-slot and a then-slot, has a bundle of slots which together specify the applicability condition, and another bundle of slots which specify the actions. In addition, there are a variety of non-executable slots that describe the heuristic, that enable its being indexed, that facilitate establishing relations between heuristics, and so on.

Lenat draws the following main conclusions about heuristics from his experiments with AM and EURISKO.

1. It is possible for a body of heuristics to guide effectively a program in searching for new concepts and hypotheses about them.

2. Heuristics must be domain-specific in order to be really effective in the discovery process.

3. Meta-heuristics (i.e., heuristics about heuristics) need not be different kinds of entities from domain-specific heuristics. They can all have the same form.

The EURISKO experiment seems to provide support to the belief that good metaheuristics, in combination with properly initialized domain-specific heuristics and domain-specific facts, would successfully enable the generation of new knowledge relating to the domain.

One catch in the above reasoning, as Lenat himself points out, is that 'observability' is a pre-requisite to effective deployment of heuristics. Experiments must be performed, data gathered, and their relevance, value or "interestingness" evaluated. A computer can currently do this only if its whole domain of interaction is fully under its experimental control. This means that, for the present, the mechanical discovery process is restricted to fields like mathematics, programming, and games, where interaction with the real-world is not an issue.

\subsection{Two kinds of knowledge revisited}

Earlier we broadly categorized knowledge into two kinds - tacit and propositionizable. So far all our discussions have been restricted to the propositionizable variety. 
What do we know about, what can we say about, tacit knowledge? The ubiquity of tacit knowledge is self-evident. Our expertise in areas like vision, speech, informal language behaviour, motor skills, is all underpinned by tacit knowledge. Our daily living is made up of behaviour in these modalities.

There have been AI efforts to build systems based on propositionized know-how to produce viable behaviour in speech, vision, and informal natural language. These attempts demonstrate how intractably complex such expert systems tend to become. And for all this complexity their achieved performance so far is extremely poor.

One can draw two alternate conclusions from such outcomes.

1. These task domains are intrinsically exceptionally complex. We have to keep trying and trying harder. Eventually we will succeed.

2. In principle, tacit knowledge cannot be 'propositionized', i.e., expertise based on the former cannot be captured in terms of the latter.

The question that remains open if we accept the second alternative is

what is the nature of tacit knowledge?

It is to be noted that in human beings and other animals, sensory-motor behaviour is underpinned by dedicated, special-purpose mechanisms - in the visual, auditory, tactile and manipulatory modalities. In this may lie a clue to finding an answer to our question. In all these modalities hard-wired programs carry out the low-level (i.e., initial stage) processing of the sensory-motor information.It has been persuasively argued that low-level processing is data driven and is not knowledge-based (Marr 1982). We can perhaps, then, argue that knowledge is tacit at this level because it is implicit in the mechanisms that support performance. Knowledge and control are not separated. Knowledge is implicit in the control structure. The mechanism itself is ultimately the representation of the expertise in these modalities.

This issue is of central importance to fifth generation computers because, as we saw in $\$ 2$, Al enters into FGC design at two levels: one in extending the interaction capabilities at the levels of vision, speech, manipulation and locomotion; and the second in extending the cognitive capabilities. If our arguments about tacit knowledge above are valid, it would seem that in order to achieve performance comparable to that of humans in the sensory-motor modalities, FGC systems must be endowed with dedicated, special-purpose peripheral mechanisms. These mechanisms must carry out the kinds of computations that the corresponding mechanisms which human beings are endowed with carry out.

\section{Concluding remarks}

We have considered in this paper how AI forms an intrinsic aspect of the competence of FGC in view of the need for them to be primarily knowledge-processing systems. Knowledge-processing arises at two levels: one in enlarging the interaction capabilities of these systems with the outside world, including human beings; and secondly, in enlarging their cognitive capabilities (i.e., in problem-solving, learning and natural language usage). We argued that there is a difference between tacit knowledge and propositionizable knowledge. Most of the current preoccupation in AI research has been with propositionizable knowledge as it relates to enlarging the cognitive capabilities of machine intelligence.

One suggestion in this paper is that tacit knowledge is tacit because it is embodied in the very mechanisms that process information, without knowledge and control being 
separated. Operations carried out by these mechanisms are data-driven as is the case with low-level sensory-motor processing in human beings. If this view is correct, then it would follow that understanding the low-level information processing aspects of the sensory-motor channels in human beings would be of great importance to finding out how to provide these kinds of competences (in vision, speech, etc.) to the FGC.

In the main body of the paper we discussed various issues that arise in knowledge representation and knowledge use. The insights that have been built up on these topics through work on expert systems were surveyed. We saw that knowledge in these taskenvironments are of two kinds: domain-specific knowledge and meta-knowledge or heuristics on how to use the first kind of knowledge, taking into account contingencies of problem-situations as they are encountered. We discussed the nature of heuristics based on the work of Lenat on AM and EURISKO.

As we pointed out earlier, the literature on AI is vast and rapidly growing. What we have been able to discuss in this paper relates only to a small set of the problems that $\mathbf{A I}$ research addresses - although it is a very important subset. There exists a considerable body of work in computer vision, speech understanding, natural language understanding and usage, all of which are of direct relevance to incorporating AI in FGC.

Even in the knowledge processing area we have considered only knowledge representation techniques that are rule-based. As we noted earlier, an alternate mode of knowledge representation is in the form of assertions, usually in the form of Horn Clauses: $P$ if $q_{1} \& q_{2} \& \ldots \& q_{n}$. Languages like PROLOG are adapted to making inferences from assertions of this type. Some of the research topics that are attracting the attention of AI workers are these: how to make inferences under incomplete information (default reasoning), under uncertainty, under temporally changing conditions, and so on. The literature on these topics is also large and rapidly growing. These areas are also of relevance to incorporating AI in FGC. The best single source of reference to work in all the areas mentioned above is the journal Artificial Intelligence.

\section{References}

Bobrow D G 1984 in Proc. Int. Conf. on Fifth Generation Computer Systems (Amsterdam: North Holland) Clarke K L, McCabe F G 1982 in Machine intelligence (eds) J E Hayes, D Michie (New York/Chichester: Halsted/Ellis Horwood) 10: $455-470$

Davis R 1977 Proc. Fifth Int. Joint Conf. on AI (Los Altos: William Kaufmann)

Duda R O, Shortliffe E H 1983 Science 220: $261-268$

Fuchi K 1984 in Proc. Int. Conf. on Fifth Generation Computer Systems (Amsterdam: North Holland)

Lenat D B 1982 Artif. Intell. 19: 189-249

Lenat D B, Brown J S 1984 Artif. Intell. 23: 269-294

Marr D 1982 Vision (San Francisco: Freeman)

Nii H P, Aiellò N 1979 Proc. Sixeh Int. Joint Conf. on Al (Los Altos: William Kaufmann)

Pople H 1977 Proc. Fifth Int. Joint Conf. on Al (Los Altos: William Kaufmann)

Ritchie G D, Hanna F K 1984 Artif. Intell. 23: 249-268

Shortliffe E H 1976 Compuler-based medical consultations: MYCIN (New York: Elsevier/North Holland)

Stefik M, Aikins J, Balzer R, Benoit J, Birnbaum L, Hays-Roth F, Sacerdoti E 1982 Artif. Intell. 18: 135173

van Melle W 1979 Proc. Sixth Int. Joint Conf. on Al (Los Altos: William Kaufmann) 